10th Intern. Conf. on Indium Phosphide and Related Materials

11-15 May 1998 Tsukuba, Japan

11:00 - 11:30

FB2-1 (Invited)

\title{
Photonic Bandgap Crystal InGaAsP Membrane Microresonators
}

\author{
A. Scherer, B. D'Urso, O. Painter, R. Lee, A. Yariv \\ Caltech, Pasadena, CA 91125
}

We have microfabricated 2-D photonic bandgap structures in a thin slab of dielectric material to define reflectors and high-Q microresonators. By selectively omitting holes from the two-dimensional photonic crystal, laser cavities and optical resonator switches can be defined. We have designed this structure with a finite difference time domain (FDTD) approach, and demonstrate some promising designs in InGaAs/InGaAsP multiple quantum well material with emission wavelength of 1.55 microns

\section{Introduction}

Fabrication of two dimensional photonic bangap crystals makes it possible to control the propagation of light within a plane. Confinement of light in the vertical direction can be attained by the use of a thin suspended membrane of high refractive index material. This waveguiding slab, which contains the 2-D photonic crystals can also be used to connect adjacent optical devices [1]. Microcavities based on photonic crystals can therefore be used to define either small laser sources or optically pumped switches. Emitted light from one or more microcavities can be used to pump others, forming a compact optical switching network. Here we design and fabricate such in-plane membrane microresonators with the purpose of connecting them in compact optical networks. Within the plane of light propagation, microcavities can be designed to interact through optical paths defined in the photonic bandgap crystals. Photonic crystals can thus provide the desired freedom in the geometrical design of the cavities as well as the coupling channels. We consider the use of a triangular array of air holes in a slab of material with a high dielectric constant [13], which has been shown to exhibit a bandgap for both TE and TM polarizations. The InGaAs/InGaAsP material system was chosen since it does not suffer from large surface recombination losses and is relatively easy to microfabricate structures into. It has previously [4] been used to demonstrate optically and electrically pumped whispering gallery mode microdisk lasers with radii down to $1 \mu \mathrm{m}$ and cavity thicknesses comparable to our designs.

\section{Procedure}

A structure with six $12 \mathrm{~nm}$ thick InGaAs quantum wells separated by five $12 \mathrm{~nm}$ thick InGaAsP barriers and InGaAsP layers on the top and bottom of the quantum wells was grown by organometallic vapor phase epitaxy (OMVPE) on an InP substrate. A surface mask composed of $60 \mathrm{~nm}$ of $\mathrm{SiO}_{2}$, followed by $5 \mathrm{~nm} \mathrm{Cr}, 50 \mathrm{~nm} \mathrm{Au}$, and 70 $\mathrm{nm}$ of polymethylmethacyclate (PMMA) was deposited on top of this sample. The PMMA resist was then exposed with a Hitachi S-4500 field-emission electron microscope to define the 2-D photonic crystals and optical cavities. After developing in cellusolve/methanol, the patterns in the PMMA layer were transferred through the $\mathrm{Cr}$ and $\mathrm{Au}$ layers with an $\mathrm{Ar}^{+}$ion beam. This metal mask was used to transfer the lithographic patterns into the $\mathrm{SiO}_{2}$ mask by using a $\mathrm{C}_{2} \mathrm{~F}_{6}$ reactive ion etching (RIE) process

[5]. Finally, the lateral resonator structures 
were etched through the InGaAs/InGaAsP multiple quantum well layer by using chemically assisted ion beam etching (CAIBE) with an argon ion beam assisted by chlorine reactive gas. After the holes were etched through the InGaAs and InGaAsP layers, the InP substrate was removed by isotropic chemical etching with $\mathrm{HCl}$ through the microfabricated holes. This procedure left a suspended membrane of perforated InGaAs/InGaAsP material to create membrane microresonators.

Triangular and hexagonal cavities were designed using the effective index of refraction of the waveguide, determined from the propagation constant of the TE mode. Next, the band structure of a 2-D photonic crystal made by etching holes into a slab of material with this index of refraction was calculated using a plane wave expansion. The band structure of a triangular array of holes was then used to design the mirrors for the triangular and hexagonal cavities such that the emission wavelength of the InGaAs was within the bandgap of the lattice. We have developed and used a finite-difference time-domain (FDTD) [6-9] method to investigate the behavior of light in membrane microresonator cavities. The steady state modes were extracted using a numerical Fourier filter.

\section{Cavity Design and Fabrication}

When taking into account the thickness of the waveguide, we determine an effective index of refraction of the waveguide layer of 2.6491 . This value can be used to calculate the band structure of a triangular array of holes etched into the material. The band structure reveals the existence of a bandgap for TM (magnetic field parallel to axis of holes), but no bandgap for TE (electric field parallel to axis of holes). Thus, the array acts as a mirror for TM polarized light but not for TE polarization. The triangular cavity is designed with normalized frequency $\omega \mathrm{a} / 2 \pi \mathrm{c}=0.31$. For the light emission peak of InGaAs at $1.55 \mu \mathrm{m}$, this requires a hole spacing $a=500 \mathrm{~nm}$ and radius $=150 \mathrm{~nm}$, which is well within the resolution range of electron beam lithography.

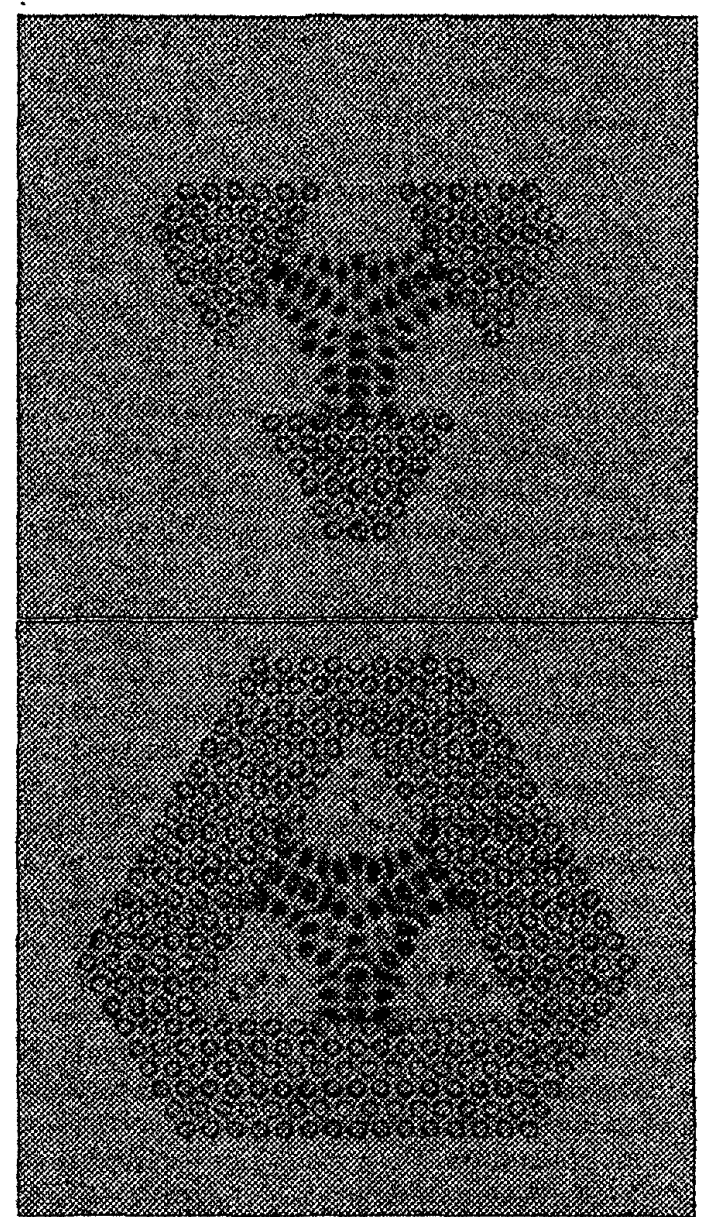

Figure 1. Calculated modes supported within thin triangular membrane cavities. Here the color indicates intensity (magnitude squared) of the magnetic field.

A triangular cavity of length $7 \mu \mathrm{m}$ per side with the mirrors described above can be modeled with the FDTD method. In order to find a mode of the full cavity 
which reflects primarily off of the middle of the sides and not out of the comers, we begin by finding a mode of the cavity without mirrors on the corners figure 1a. The solution for this cavity is then used as the initial field for filtering in the complete cavity figure $1 \mathrm{~b}$. Notice that the mode interacts strongly with the center of each side and only weakly with the corners, as desired

The quality (Q) of these modes is also calculated with a two-dimensional FDTD method. The full triangular cavity without any output channels has a $Q$ of 4300. The cavity $Q$ for this mode is substantially reduced to a value of 900 when a coupling waveguide is included through the side of the triangular cavity which reflects a high field intensity. Examples of microfabricated hexagonal and triangular cavities are shown in Figure 2 , where a scanning electron micrograph shows the geometry of these membrane microresonators. The distances between holes in the $2 \mathrm{D}$ photonic bandgap mirrors correspond to the optimized dimensions calculated earlier.

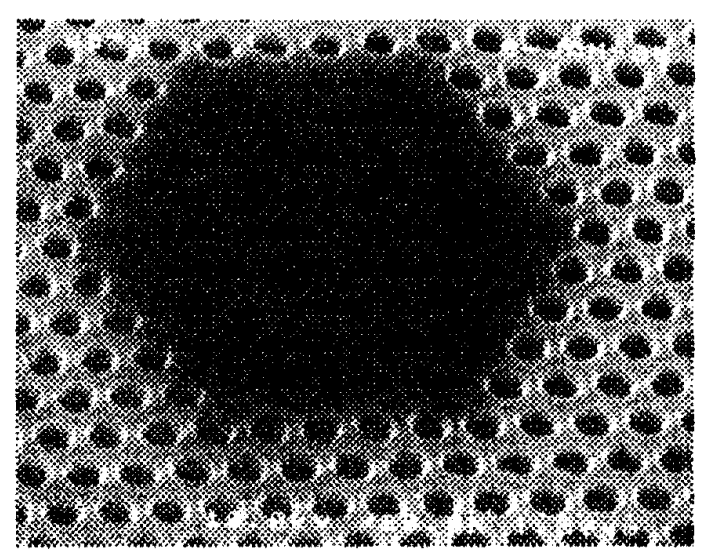

Figure 2. Figure 2a-c. SEM micrographs showing examples of microfabricated hexagonal and triangular optical micromembrane cavities with $\sim 5 \mu m$ lateral dimensions. Figure $2 c$ shows a cavity defined by a single defect, with the smallest cavity volume. The InGaAsP membrane contains five InGaAs quantum wells and is approximately $160 \mathrm{~nm}$ thick.
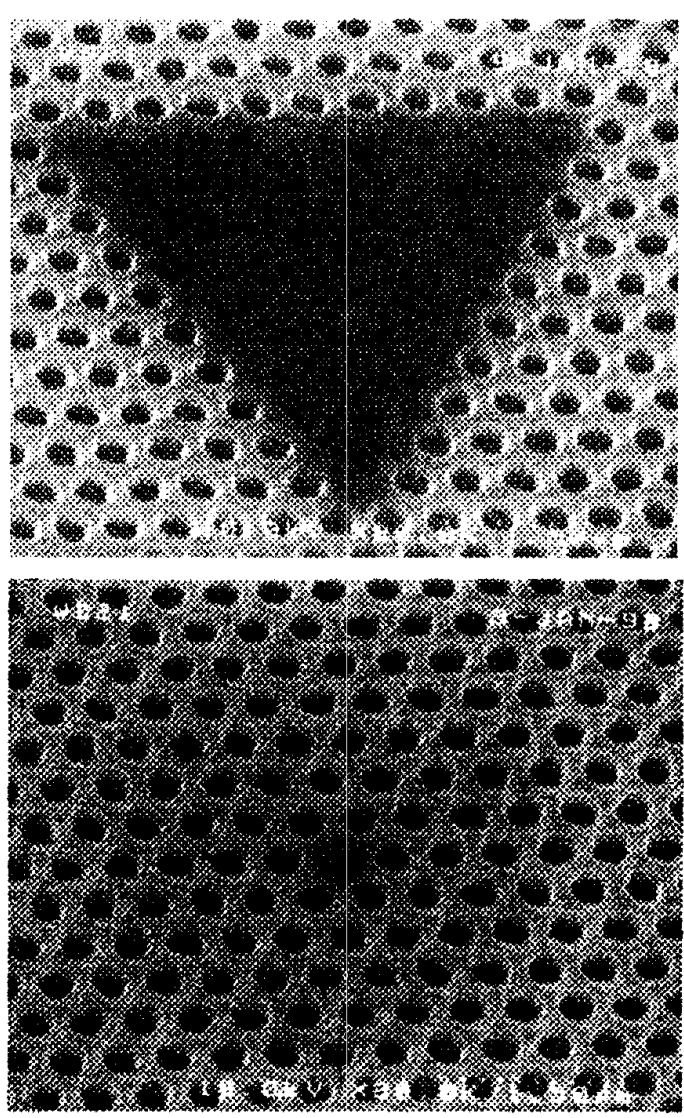

Some of the smallest optical cavities which can be defined within the two-dimensional photonic crystals consist of single defects which support only a few optical modes. Single defect optical microcavities have the potential of altering the spontaneous emission rate of light. The modes which are supported by these optical cavities can be pumped more efficiently. We are currently measuring the performance of single defect membrane microresonators (Fig $2 \mathrm{c}$ ) by experimentally determining their $Q s$ and luminescence efficiencies.

We have shown that photonic crystals can be used to localize light into a 
triangular cavity. In particular, we have designed a mirror and cavity for use with the InGaAs/InGaAsP system. We have also demonstrated the the fabrication of the triangular, hexagonal and single defect cavities which were designed and modeled. The fabrication sequence used is summarized in Figure 3. Presently, these structures are evaluated by optical pumping.

\section{References}

1. J.D. Joannopolous, R.D. Meade, J.N. Winn, Photonic Crystals, (Princeton University Press, 41 William Street, Princeton, NJ 08540, 1995)

2. S. Fan, P.R. Villeneuve, J.D. Joannopoulos, E.F. Schubert, Proc. Photonics West'97
3. T.F. Krauss, R.M. De La Rue, Appl. Phys. Lett., 68, 1613, (1996).

4. A. Levy, R. Slusher, Appl. Phys. Lett., (1992)

5. J. O'Brien, O. Painter, C.C. Cheng, R.

Lee, A. Yariv, A. Scherer, Electronics Lett., 32, 2243, (1996)

6. M. Kanskar, P. Paddon, V. Pacradoui, R. Morin, A. Bush, J.F. Young, S.R. Johnson, J. Mackenzie, T. Tiedje, Appl. Phys. Lett., 70, 1438, (1997)

7. M. Bartsch et al., Computer Physics Communications, 72, 22 (1992)

8. D.H. Choi, W.J.R. Hoefer, IEEE Transactions on Microwave Theory and Techniques, 34, 1464, (1986)

9. Z. Bi, Y. Shen, K. Wu, J. Litva, IEEE Transactions on Microwave Theory and Techniques, 40, 1611

(1992)

Figure 3. Schematic process sequence used to define the micro-membrane devices. InP is used as a substrate and is selectively attacked by a hydrochloric acid etch to define the final freestanding membrane. The mask layer consists of a silicon dioxide etch mask and PMMA e-beam resist.
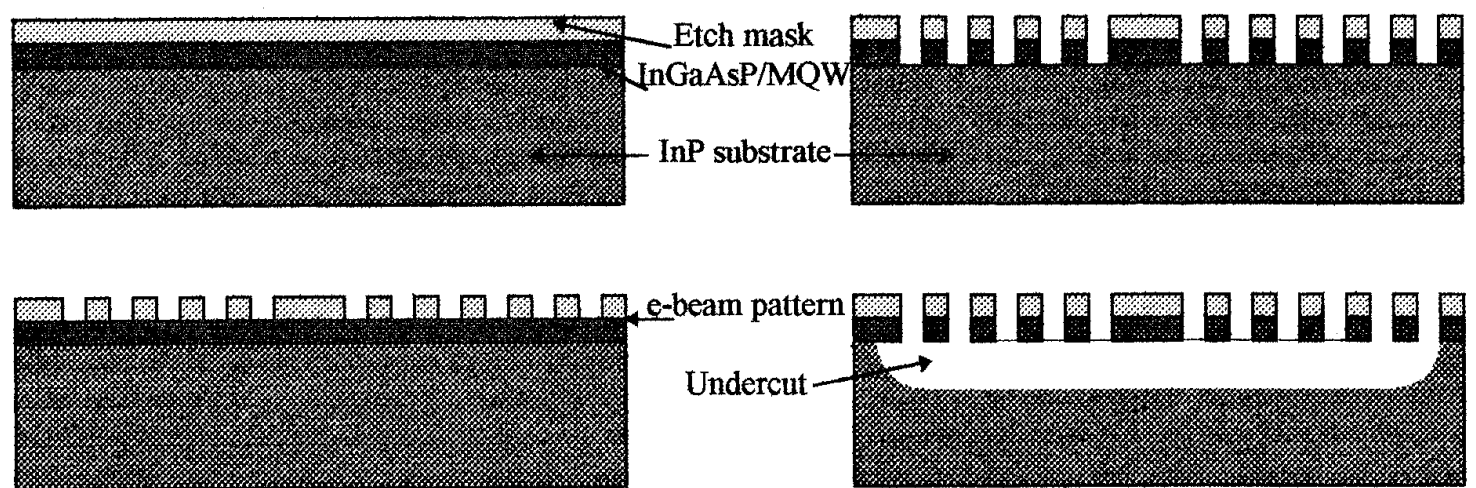